\title{
On polar daily geomagnetic variation
}

\author{
Paola De Michelis ${ }^{1,}$, Giuseppe Consolini ${ }^{2}$ \\ ${ }^{1}$ Istituto Nazionale di Geofisica e Vulcanologia, Rome, Italy \\ ${ }^{2}$ INAF - Istituto di Astrofisica e Planetologia Spaziali, Rome, Italy
}

\author{
Article history \\ Received May 27, 2015; accepted October 5, 2015. \\ Subject classification: \\ Daily geomagnetic variation, Sun-Earth interaction, Natural orthogonal component analysis.
}

\begin{abstract}
The aim of this work is to investigate the nature of the daily magnetic field perturbations produced by ionospheric and magnetospheric currents at high latitudes. We analyse the hourly means of the X and $\mathrm{Y}$ geomagnetic field components recorded by a meridian chain of permanent geomagnetic observatories in the polar region of the Northern Hemisphere during a period of four years (1995-1998) around the solar minimum. We apply a mathematical method, known as natural orthogonal component (NOC), which is capable of characterizing the dominant modes of the geomagnetic field daily variability through a set of empirical orthogonal functions (EOFs). Using the first two modes we reconstruct a two-dimensional equivalent current representation of the ionospheric electric currents, which contribute substantially to the geomagnetic daily variations. The obtained current structures resemble the equivalent current patterns of DP2 and DP1. We characterize these currents by studying their evolution with the geomagnetic activity level and by analysing their dependence on the interplanetary magnetic field. The obtained results support the idea of a coexistence of two main processes during all analysed period although one of them, the directly driven process, represents the dominant component of the geomagnetic daily variation.
\end{abstract}

\section{Introduction}

The geomagnetic field, as observed at the Earth's surface, changes continuously with time. Changes of very short duration (from seconds to days) are the magnetic manifestation of an increased level of the solar wind-magnetosphere interaction, which is responsible for the enhancement of the intensity of electric current systems circulating in the magnetosphere and ionosphere. These electric current systems involve the generation of additional magnetic fields, which are recorded on the ground as variations superimposed on the main field. One of the most important purposes of the geomagnetism is to examine the configuration of these electric current systems and to elucidate their driving processes in terms of the solar wind-magnetosphere interaction.
Among the possible short-term geomagnetic variations the smoothed and most regular one is that observed on magnetically quiet days, which is known as solar quiet daily geomagnetic field variation $\left(S_{q}\right)$. At low and mid-latitudes this variation mainly arises from the ionospheric current system flowing in the so-called dynamo region. This current system can be well approximated by a 2-D current flowing in the ionospheric E-region between 90 and $130 \mathrm{~km}$ and consists of two large current vortices circulating about foci at $\pm 30^{\circ}$ geomagnetic latitude. Viewed from the Sun, circulation is counterclockwise in the Northern Hemisphere and clockwise in the Southern Hemisphere. The $S_{q}$ current is dominant in the daytime ionosphere where ionospheric conductivity is relatively large, and it is driven by electric fields originating from the ionospheric dynamo via the interaction between ionized and neutral particles. This regular variation depends on the local time, latitude, season and solar cycle [Campbell 1989, Richmond 1995, Olsen 1997]. In contrast, at high latitudes, a particular type of daily geomagnetic variation exists which differs from what one would expect from the dynamo theory of daily geomagnetic variation. According to Nagata and Kokubun [1962], Kawasaki and Akasofu [1967] and Feldestein and Zaitzev [1967], the geomagnetic daily variation field in the polar caps on geomagnetically quiet days consists not only of the well established $S_{q}^{o}$ field, which is a smooth extrapolation to the polar region of $S_{q}$ defined at mid and low latitudes, but also of an additional field, the $S_{q}^{p}$ field. It is mainly generated by a current system limited to latitudes above $60^{\circ}$ and consists of two current vortices confined to the polar caps, with their foci located near the dawn and dusk meridians. The pattern of this current system is partially modified by the processes that happen in the magnetosphere and by its interaction with the interplanetary magnetic field (IMF). Indeed, the currents flowing in 
and near the polar cusps and in the dayside polar caps are coupled, through the field-aligned currents, with the magnetospheric currents. It is for this reason that the $S_{q}^{p}$ current system is strongly enhanced and modified during geomagnetic disturbances and auroral substorms when the effects of non-vanishing transverse IMF components are superimposed on the quiet time configuration of the current system. However, the existence of a twin vortex system above $70^{\circ}$ of latitude for all orientations of the IMF, indicates that some elements of the current pattern may be independent of the IMF [Friis-Christensen et al. 1985]. This current system portion is the real quiet part of the polar equivalent current system. However, quiet days are quite rare events in the polar regions because the geomagnetic field is disturbed at high latitudes by the continuous transfer of energy from the solar wind to the magnetosphere, which occurs even during quiet conditions.

We emphasize that it is not simple to extrapolate the geomagnetic solar quiet daily variation from the daily record of geomagnetic variations because other current systems produced by different sources, external as well as internal to the Earth, are responsible for the creation of magnetic fields that overlap each other. Indeed, ground magnetometers are capable of detecting fields due to distant magnetospheric sources such as the magnetotail current, the partial ring current, the substorm current wedge and the dayside current wedge and their contributions can be not entirely negligible. To sort out these single contributions can be extremely difficult but the knowledge of the different sources, which contribute to the daily variation of the geomagnetic field, can be the key to investigate the solar windmagnetosphere-ionosphere coupling from a different point of view.

$\mathrm{Xu}$ and Kamide [2004] and Chen et al. [2007] applied the natural orthogonal component (NOC) analysis to separate and recognize the different contributions of the ionospheric-magnetospheric current systems to the daily changes of the geomagnetic field as recorded at mid-latitude ground-based geomagnetic observatories. Successively, De Michelis et al. [2009] extended these works at high latitudes by analysing the hourly values of the three magnetic field components $(X, Y$ and $Z$ ) recorded at the Italian geomagnetic observatory TNB located in Antarctica during a period of 4 years (1995-1998).

Here, using magnetic field data coming from a meridian chain of permanent geomagnetic observatories in the Northern Hemisphere, we identify the different sources that contribute to the daily variation of the Earth's magnetic field at high latitude by applying the NOC analysis. The aim is to reconstruct the spatial distribution of those current systems, which are mainly responsible for the geomagnetic daily variation at high latitude and to analyse their changes with the geomagnetic activity level and the interplanetary magnetic field conditions.

The paper is organized as follows. At first, the data sources are discussed then a brief summary of NOC analysis is presented. Following this, the NOC analysis is applied on the selected geomagnetic time series. Finally, the implications of findings are discussed.

\section{Data}

To study the geomagnetic field variation in the polar region of the Northern Hemisphere we use the hourly mean values of the $X$ and $Y$ components of the geomagnetic field recorded during four years (1995-1998) at six permanent geomagnetic observatories characterized by the same magnetic longitudinal position and latitudinal values ranging from $62^{\circ}$ to $83^{\circ} \mathrm{N}$. We make use of recordings only obtained by permanent observatories fulfilling international standards. All these observatories are part of the worldwide network of observatories known as INTERMAGNET. The high data quality, especially a good stability of instruments, guarantees that our objective can be reached. The selected network of geomagnetic observatories is reported in Table 1. It provides the geographic and corrected geomagnetic co-

\begin{tabular}{ccccccc}
\hline IAGA code & Lat & Long & Mlat & Mlong & MLT & L-shell \\
\hline BLC & 64.32 & 263.99 & 73.92 & 327.50 & 6.84 & 13.08 \\
CBB & 69.12 & 254.97 & 77.25 & 308.85 & 7.93 & $\infty$ \\
FCC & 58.76 & 265.91 & 68.92 & 332.25 & 6.56 & 7.75 \\
MEA & 54.62 & 246.65 & 62.08 & 305.70 & 8.17 & 4.58 \\
RES & 74.69 & 265.12 & 83.51 & 319.07 & 7.30 & $\infty$ \\
YKC & 62.48 & 245.52 & 69.50 & 300.48 & 8.49 & 8.18 \\
\hline
\end{tabular}

Table 1. List of geomagnetic observatories selected in this work. Observatories are identified using their IAGA code. Geographyical and corrected magnetic coordinates are given in degrees. MLT is given in UT (hours) at time when given point is at midnight. L-shell is given in Earth's radii $R_{E}$. The geomagnetic coordinates were calculated for 1996.0 using the routine available at http:/ / modelweb.gsfc.nasa.gov. 
ordinates of the geomagnetic observatories together with the magnetic local time (MLT) respect to universal time (UT) that we have obtained using NASA service http:/ / omniweb.gsfc.nasa.gov/vitmo/cgm_vitmo.html.

We use the $X$ and $Y$ components instead of $H$ and $D$ components of the Earth's magnetic field because of the reconstruction of the equivalent current systems is generally obtained from the $X, Y$ and $Z$ magnetic field values. It is known that there is a considerable deviation between the direction of the $H$ component (magnetic North) and the $X$ component (geographic North) especially at the higher-latitudes observatories. At high latitudes the magnetic perturbation in the geographic North component depends almost equally on the magnetic perturbation along $H$ and $D$ and even the sign of the magnetic perturbation along $X$ can be opposite to the sign of the perturbation along $H$. For this reason, to compare our results with those obtained using different methods of analysis, it is important to use the same reference system.

\section{Method of analysis: natural orthogonal compo- nent}

To identify the different current systems, which contribute to the observed geomagnetic variations on the ground, we apply the NOC method, which permits us to extract those structures that remain coherent throughout a time series. In practice, a set of orthogonal eigenvectors and eigenvalues is estimated from observed data. By combining these eigenvectors the observed variables can be written in terms of the smallest possible set of natural orthogonal basis functions. The core of this method is described in detail in Langel [1987], and its application to geomagnetic data is widely discussed in literature. Frynberg [1975], Pushkov et al. [1976] and Rotanova et al. [1982], for example, used the NOC analysis to separate the geomagnetic field into longer- and shorter-wavelength components while Golovkov et al. [1978], Xu et al. [1990] and De Michelis et al. [2009, 2010] used it to separate magnetic variations into quiet and disturbed components at different latitudinal values. At the end, the same method was applied by Sun et al. [1998, 2000] to separate the substorm current system into directly driven and loading-unloading components and by Consolini et al. [2009] to study the origin of the dynamical complexity in the solar cycle.

Before describing the application of NOC analysis to our data, we introduce this method briefly. We consider a variable $x\left(d_{i}, t_{j}\right)$ representing the value of a magnetic field component $\left(X\right.$ or $Y$ ) for a certain day $d_{i}$, and time $t_{j}$. Given a number of samples of $x\left(d_{i}, t_{j}\right)$ at different days and times, the application of the NOC method allows us to extract a smaller set of variables, lets say of empirical orthogonal functions (EOFs) and principal components (PCs), capable of describing the whole set of observations. Therefore, we can decompose the daily variation of any geomagnetic component in terms of a basis of empirical orthogonal functions (EOFs) $\varphi^{k}\left(t_{j}\right)$ :

$$
x\left(d_{i}, t_{j}\right)=\sum_{k=1}^{N} A^{k}\left(d_{i}\right) \varphi^{k}\left(t_{j}\right)
$$

where the collection of values $x\left(d_{i}, t_{j}\right)$ are the elements $x_{i j}$ of the matrix $X(m \times n)$ whose rows correspond to the days $(i=1,2, \ldots, m)$ and columns correspond to the time $(j=1,2, \ldots, n), N$ is the number of components chosen for the decomposition (i.e. the truncation level), $\varphi^{k}\left(t_{j}\right)$ is the mode (EOF) of the $k$-th component describing the daily variation (i.e. it is the basis used for the expansion), $A^{k}\left(d_{i}\right)$ is the principal component (PC) and represents the amplitude of $k$-th mode. To evaluate the EOFs and the associated PCs from a dataset the error, made in the representation of the observed data by means of the expansion (1), has to be minimized. This error depends on the truncation level $N$ : the higher the truncation level, the lower the error.

Since we are looking for a number of components capable of describing completely the observed variable, one additional assumption is that the $\operatorname{EOFs}\left(\varphi^{k}\right)$ are orthogonal and the PCs $\left(A^{k}\right)$ vary independently. This leads to solve the well-known eigenvalue-eigenvector problem that permits us to estimate the eigenvalues $l_{k}$ together with the corresponding eigenvectors $\varphi^{k}$ and amplitudes $A^{k}$ for $k=1, \ldots, N$ (see for more details Xu and Kamide [2004]). Once we obtain the EOFs $\left(\varphi^{k}\right)$, the PCs $\left(A^{k}\right)$ can be evaluated by simply projecting the data along each EOFs.

It is consequently evident that the NOC method supplies a different way to create the orthogonal function system. In this method the empirical orthogonal functions are not artificially chosen in advance, but are determined together with the principal components using observed data. This method represents a radical departure from all the classical methods and being the expansion basis derived from the data it is a posteriori, and highly efficient method. However, the obtained basic functions reflect the characteristics of the analysed data, and are closely related with their origin.

\section{Results and discussion}

As already mentioned in the previous section, we use the hourly average variations of the $X$ and $Y$ magnetic field components recorded at the geomagnetic observatories reported in Table 1 from January 1, 1995, to December 31, 1998. This period is characterized by a very low solar activity being the minimum level of 
solar activity between cycles 22 and 23 occurred in May of 1996. For this reason the selected period may be a good choice to reveal those patterns within the complex geomagnetic variation, which are a basic characteristic of the daily variation. We apply the NOC analysis to all our selected observatories. Looking at the spectra of the eigenvalues (which provide a measure of the root-mean-square values of the corresponding PC) for the $X$ and $Y$ magnetic field components recorded at the different observatories (data are not shown), we notice that they are all characterized by a rapid drop at small $k(k<5)$. Consequently, we assume that most of the variability observed in the geomagnetic field daily variation can be explained in terms of a very small number of natural components, which are sufficient to capture most of the properties of the observed variable. The rest of the eigenvalues can either take into account transient fluctuations and noise or represent the contribution of stochastic processes. For this reason, we consider only the first two natural components, which dominate over the rest. As example of our results we report the first two EOFs obtained by analysing the $X$ and $Y$ components recorded at RES geomagnetic observatory in Figure 1, while the associated PCs in Figure 2.

Using the first EOFs associated with the $X$ and $Y$ components of each observatory, we plot the horizontal magnetic disturbance vectors in geomagnetic latitude and MLT coordinates. The corresponding equivalent current vector plot is constructed by rotating the horizontal disturbance vectors clockwise by $90^{\circ}$. The obtained equivalent current vector plot is shown in Figure 3 where the vector length gives us an estimation of the current intensity. This current intensity is determined

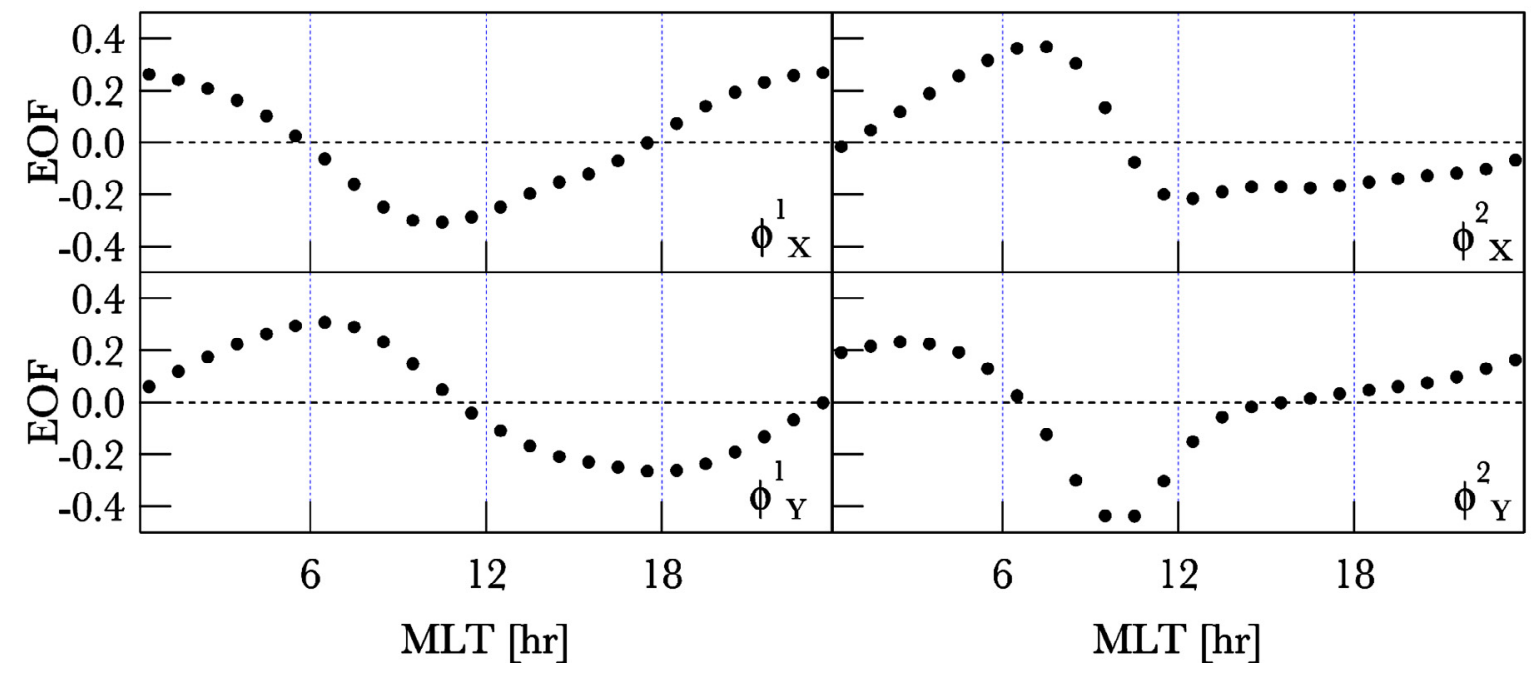

Figure 1. On the left: the first EOFs for $X$ and $Y$ magnetic field components. On the rigth: the second EOFs for $X$ and $Y$ magnetic field components. The results are relative to RES geomagnetic observatory.

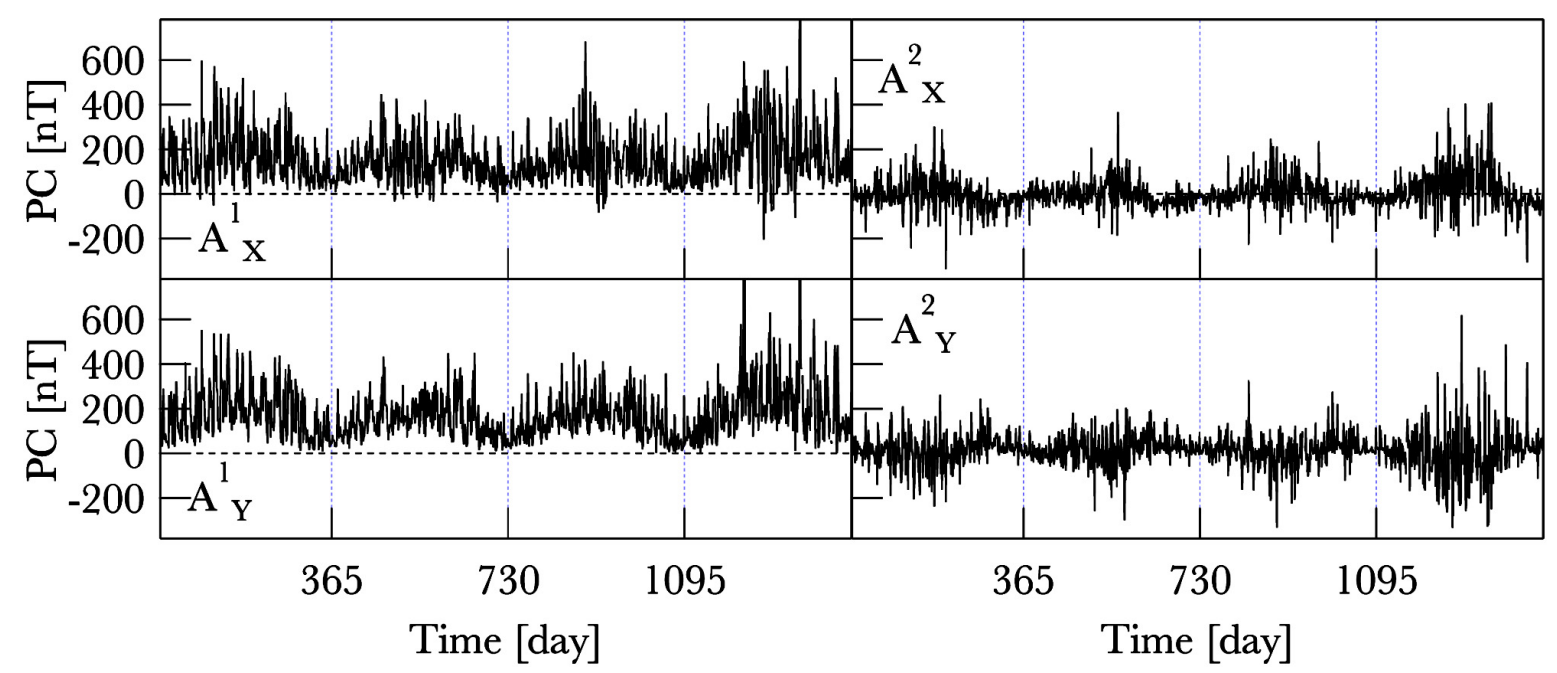

Figure 2. On the left: the PCs corresponding to the EOFs reported on the left in Figure 1. On the rigth: the PCs corresponding to the EOFs reported on the rigth in Figure 1. 
considering, for each observatory, the average values of the amplitude (PC) associated with each EOF evaluated over all analysed period. On the top panel of Figure 3 we report the distribution of the equivalent current vectors in the Northern Hemisphere obtained by considering the first EOFs. On the bottom panel of the same figure we report the distribution of the equivalent current vectors obtained in the case of the second EOFs.

The distribution of the equivalent current vectors obtained considering the first EOFs (reported on the top panel of Figure 3) shows clearly a two-cell convection pattern. These two cells are located in the 12-18 MLT sector and 21-03 MLT one, respectively. This result is well in agreement with the structure of the $S_{q}^{p}$ current system, which is formed by two vortices where the current flows in opposite directions. The temporal trends of the amplitudes associated with these EOFs display a seasonal trend characterized by a maximum in the boreal summer and a minimum in the winter. Figure 4 shows an example of this trend for the amplitude of the current vector associated with the first EOFs in the case of CBB geomagnetic observatory. This seasonal trend is well in agreement with the characteristic of the $S_{q}^{p}$ field intensity, which shows a strong seasonal variation. Anyhow, this average current pattern characterized by a two-cell convection pattern is also similar to that obtained by Akasofu et al. [1983] and by Sun et al. [1998] resembling the equivalent current pattern of DP2, which enhances during the growth phase and the expansion phase of substorms and decays during the recovery one. The two current systems (Hall current and DP2) are actually very similar to each other, the only essential difference between them is that the DP2 current system extends at lower latitudinal values than the Hall current one.

To study the possible dependence of this current system on the geomagnetic activity level, we repeat our analysis considering two different datasets. We select

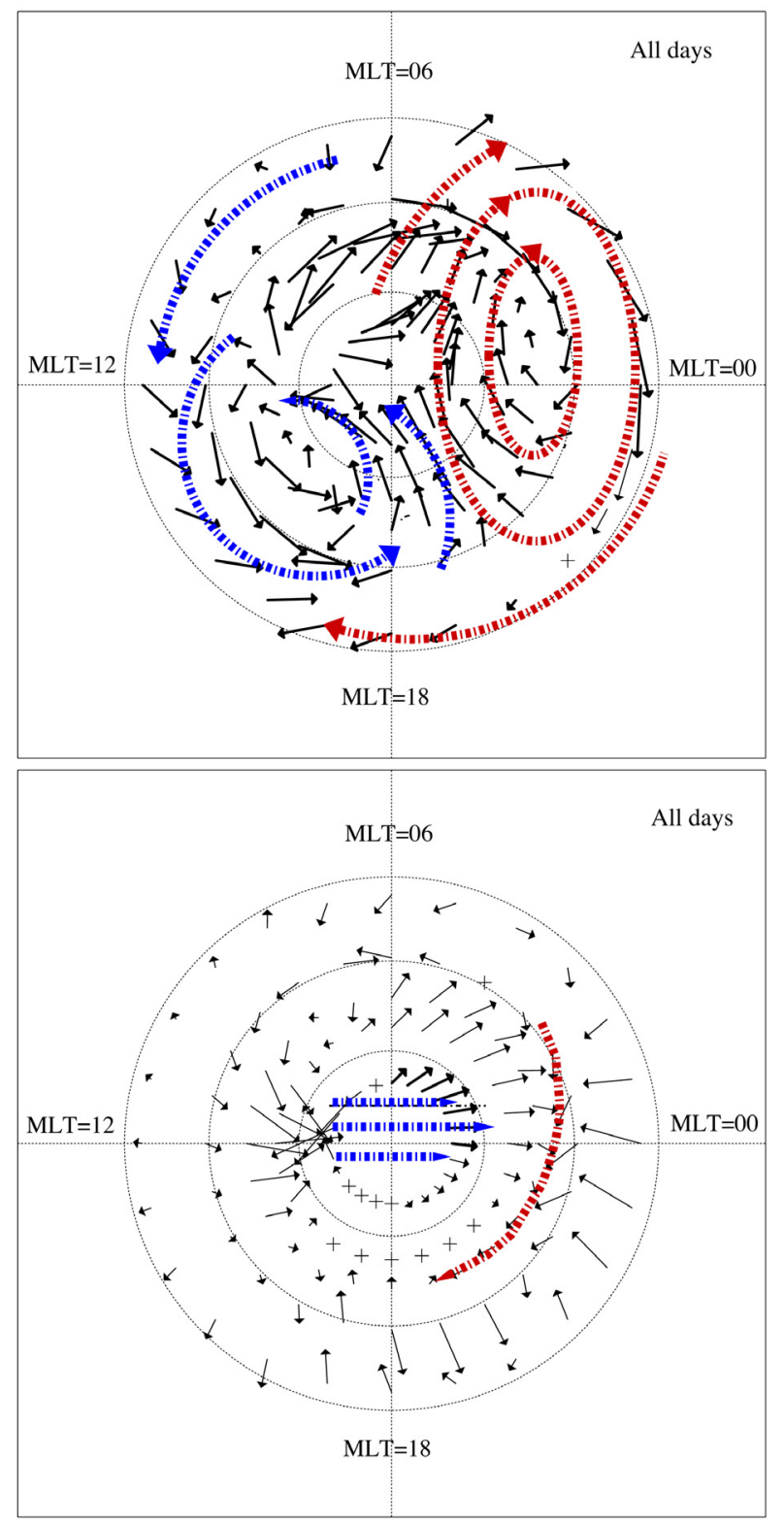

Figure 3. Polar diagrams of equivalent current vectors in the Northern Hemisphere. Coordinates are magnetic latitude from $60^{\circ}$ to the North magnetic pole, and MLT. The equivalent current vectors are calculated by means of the first EOFs on the top panel and the second EOFs on the bottom panel. The three circles mark, from the outermost one, the latitude of $60^{\circ}, 70^{\circ}$, and $80^{\circ} \mathrm{N}$, respectively.

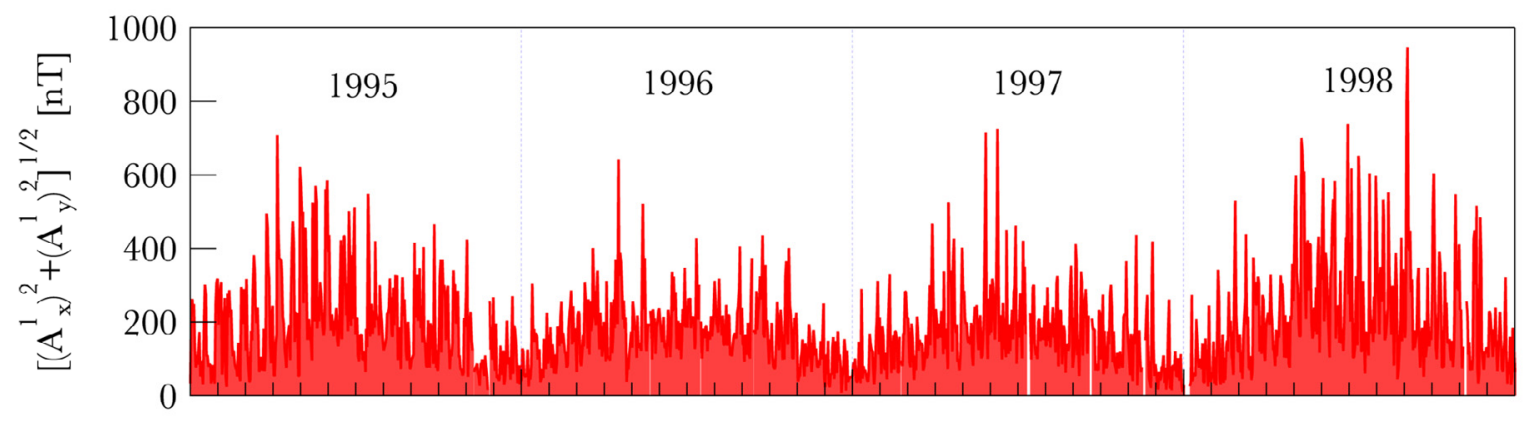

Time [day]

Figure 4. Temporal trend of the amplitude of the current vector associated with the first EOF in the case of CBB geomagnetic observatory. 

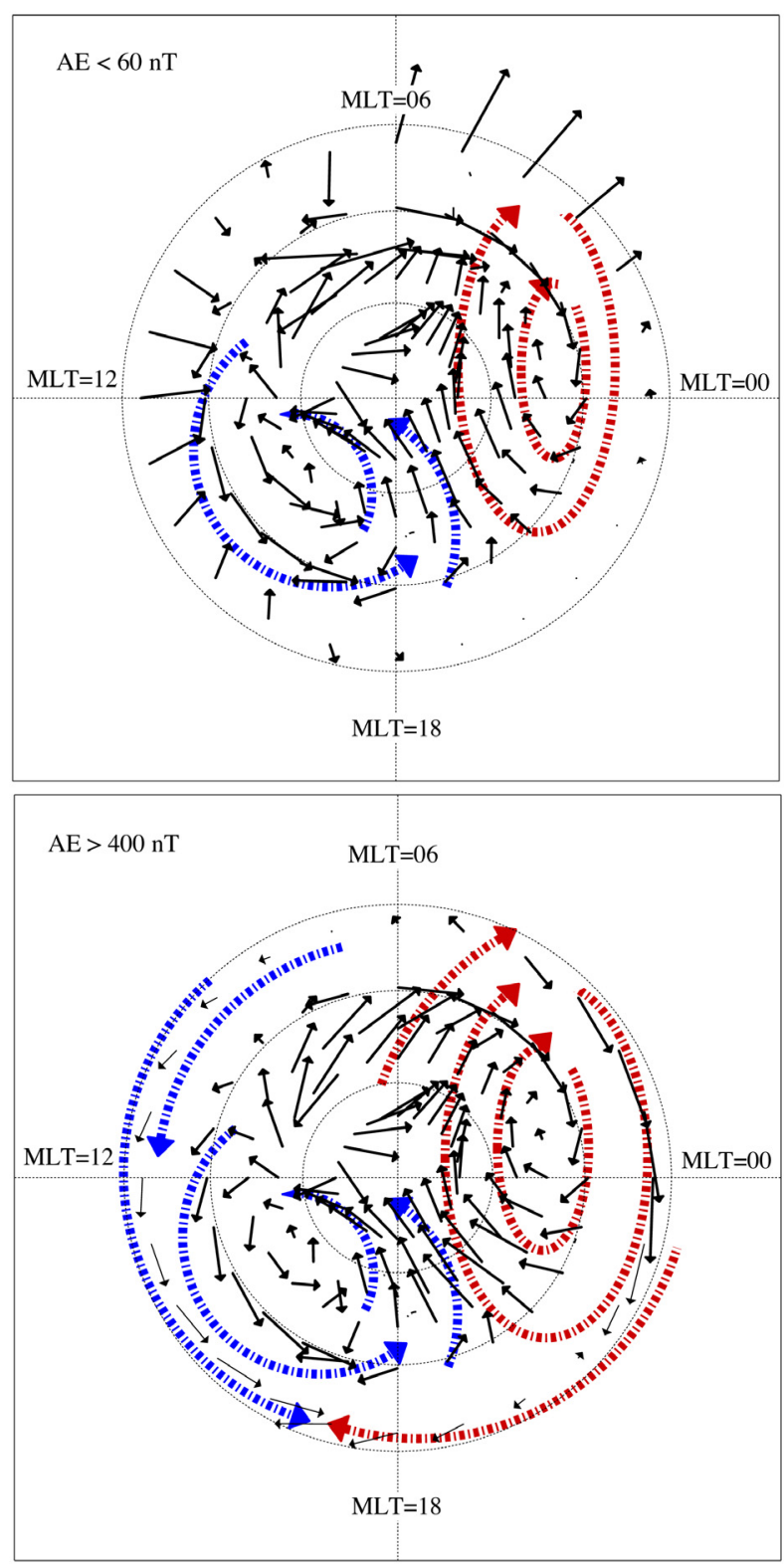

Figure 5. Polar diagrams of equivalent current vectors in the Northern Hemisphere. Coordinates are magnetic latitude from $60^{\circ}$ to the North magnetic pole, and MLT. The equivalent current vectors are calculated by means of the first EOFs on the top panel in the case of a quiet period $(A E<60 \mathrm{nT})$ and a disturbed one $(A E>$ $600 \mathrm{nT}$ ) on the bottom panel. The three circles mark, from the outermost one, the latitude of $60^{\circ}, 70^{\circ}$, and $80^{\circ}$, respectively.

days characterized by both a low geomagnetic activity level and a high one. To select the geomagnetic activity level we consider the Auroral Electrojet $(A E)$ geomagnetic index. This index is obtained from a number (usually greater than 10) of stations distributed in local time and in the latitude region that is typical of the Northern Hemisphere auroral zone [Davis and Sugiura 1966]. It is designed to provide a global, quantitative measure of auroral zone magnetic activity produced by enhanced ionospheric currents flowing below and within the auroral oval. As representative of a low geomagnetic activity level we select the days characterized by
$A E<60 \mathrm{nT}$ while in the case of high activity level we consider values of $A E>400 \mathrm{nT}$. The structure of the equivalent current vectors obtained from the two different magnetic disturbance levels is very similar. It always shows a two-cell convection pattern. The only difference between the two current structures (Figure 5 ) is a major expansion of the vortices towards lower latitudinal values in the case of high geomagnetic activity level. This behaviour is typical of the DP2 current system which consists of two ionospheric current vortices (within each hemisphere) that extend to mid-latitude and their effects are observed outside of the polar region during geomagnetically disturbed periods.

The distribution of the equivalent current vectors obtained taking into account the second EOFs (reported on the bottom panel of Figure 3) exhibits a more complex structure than that obtained from the first EOFs. We have: an anticlockwise vortex in the morning sector, an antisunward component in the polar cap and a westward electrojet around $70^{\circ}$ of latitude in the morning-midnight sector. This last current system, which seems quite stable with the change of geomagnetic activity level, recalls the DP1 current system. The DP1 current system, which is linked to the occurrence of geomagnetic substorms, consists essentially of a strong auroral electrojet that rotates westward and develops within a longitudinal band limited in the midnight sector.

To characterize better the distribution obtained by the equivalent current vectors, we analyze how the current vector amplitudes depend on the interplanetary magnetic field. We consider the daily values of the $B_{y}$ and $B_{z}$ component of the interplanetary magnetic field (IMF) in the GSM system in the period between 1995 and 1998 and for each selected geomagnetic observatories we analyze how the current amplitude module, obtained by considering separately the first and the second EOFs evaluated over the whole selected period, depends on the interplanetary magnetic field. Figure 6 exhibits the obtained results in the case of the first EOFs. For each selected geomagnetic observatories, we have reported the values of the current amplitude module as a function of the $B_{y}$ and $B_{z}$ values. They are three-dimensional plots where the colour from white-pale pink to dark red-black describes the current intensity. Data reported in this figure show the current amplitude increases enormously when $B_{z}<0$ regardless of the sign and the value of $B_{y}$. This result confirms the direct interaction of the current system with the solar wind suggesting that the distribution of the equivalent current vectors obtained considering the first EOFs describes the DP2 current system. According to Clauer and Kamide [1985] the 


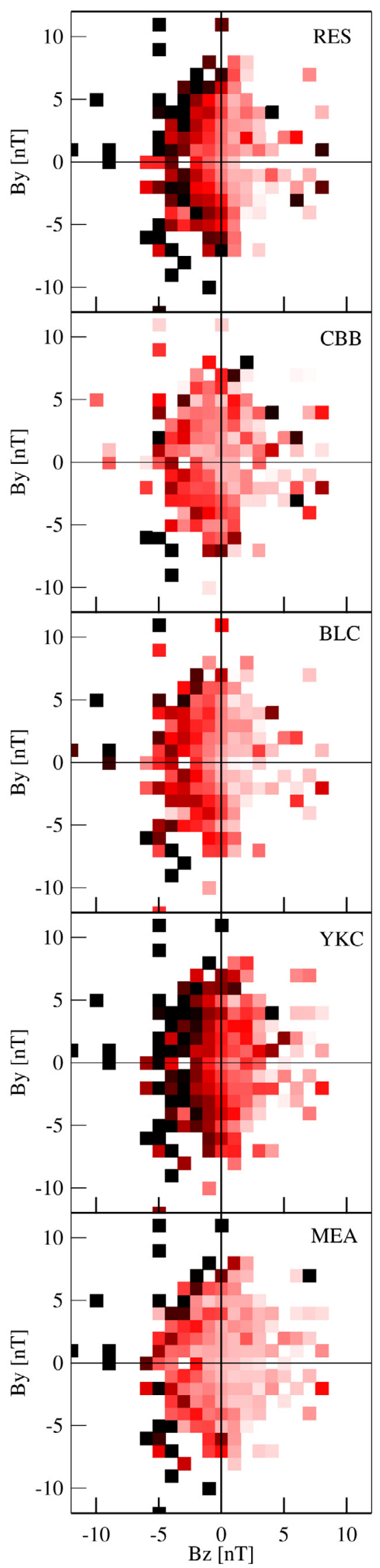

Figure 6. The amplitude module of the equivalent current vectors calculated by means of the first mode as a function of the $B_{y}$ and $B_{z}$ values for each geomagnetic observatory considered in this study.

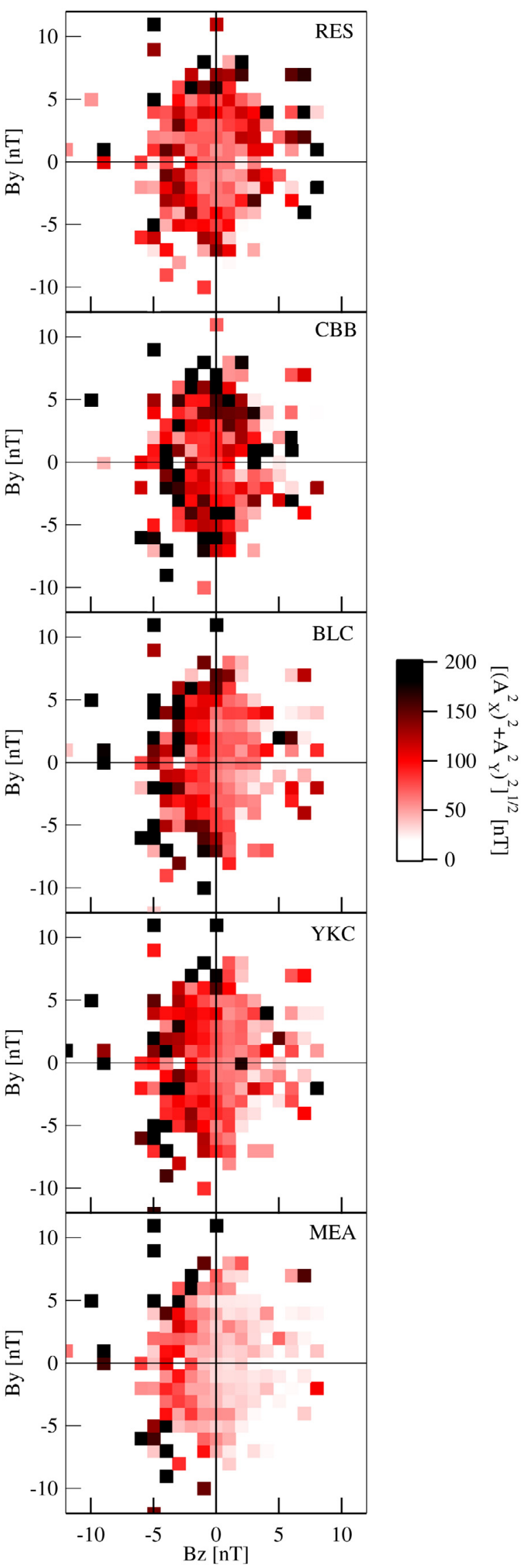

Figure 7. The amplitude module of the equivalent current vectors calculated by means of the second mode as a function of the $B_{y}$ and $B_{z}$ values for each geomagnetic observatory considered in this study (note the change in scale). 
fluctuations of the DP2 pattern show a high degree of correlation with those of IMF north-south component. This suggests that the DP2 pattern reflects the convection driven by electric fields, which exist within the magnetosphere as a result of the direct interaction between the solar wind and magnetosphere.

Figure 7 displays the results obtained by applying the same analysis to the second current system. The results achieved are slightly different from what is obtained and displayed in Figure 6. The correlation between the current amplitude and the interplanetary magnetic field exists only outside the polar cap where the westward auroral electrojet flows. This current is connected to the asymmetric ring current through field-aligned currents that flow downward near noon and upward in the evening sector. This equivalent three dimensional current system intensifies during substorms. This is the current which depends on the $B_{z}$ magnetic field component.

\section{Summary and conclusions}

Since the magnetic data obtained at the Earth's surface include valuable information about a variety of current sources flowing in the ionosphere, magnetosphere, at the magnetopause, and within the Earth's interior the ground magnetic perturbations can be used to study magnetospheric and ionospheric dynamics. An interesting problem to solve is the relationship between the ionospheric currents and the magnetic fields generated by them and the estimation of the relative importance of these currents in producing global or local magnetic perturbation patterns. We have tried to overcome this problem using the NOC analysis. By investigating the nature of the geomagnetic daily variations recorded at several geomagnetic observatories along magnetic meridian in the North polar region, we have found that the main contribution to the polar daily variation for $X$ and $Y$ magnetic field components comes from two different current systems. One consists of two-cell convection pattern, a clockwise cell in the evening sector and a counter-clockwise cell in the morning one, the other is a westward electrojet around the midnight sector between $65^{\circ}$ and $70^{\circ}$ latitude, an antisunward current in the central polar cap, and a sunward current at lower latitudes, both on the dawn and dusk sides. Thus, the patterns of the first two natural components resemble the equivalent current patterns of DP2 and DP1, respectively. Since the magnetic data are known to be representative of specific states of the magnetosphere controlled by the IMF, we have also analysed the dependence of intensity of these equivalent current systems on the interplanetary magnetic field. We have found that the DP1 variation, while showing a general dependence on the southward interplanetary magnetic field $B_{z}$ component, does not exhibit as strong one-toone correspondence as does DP2, suggesting that the DP1 disturbance is modulated by internal magnetospheric processes. These results have been obtained for a period around a minimum of solar activity (the lowest number of sunspot cycle 22 was officially recorded in May 1996) and consequently it is reasonable to extend them also to the years characterized by a stronger high solar activity level.

The obtained results are well in agreement with those obtained by Sun et al. [1998], which used magnetic records collected during the International Magnetospheric Study (IMS) from a total of 71 stations during March 17-19, 1978 with a time resolution of 5 min. By applying the method of natural orthogonal component, they showed that the first natural component and the second one were dominant and exhibited the well-known characteristics of the directly driven component and unloading component, respectively. Furthermore, Sun et al. [1998]'s analysis showed that the directly driven component tended to dominate over the unloading component, except for a brief period soon after substorm onset. In our study we have considered a much longer period than that used by Sun et al. [1998] (4 years instead of 3 days) and the obtained results confirm that the distribution of the equivalent current vectors calculated by means of the first EOFs is a direct consequence of the directly driven process where a portion of the energy entering the magnetosphere is dissipated within the magnetosphere-ionosphere system. Differently, the distribution of the equivalent current vectors calcualted by means of the second EOFs is a direct consequence of the so-called loading-unloading process, which involves the storage of the remaining energy input in the magnetic field and plasma of the magnetotail with the next deposition of the energy stored into the auroral ionosphere and into the ring current. Consequently, our results confirm that two different processes (driven directly process and loading-unloading process) coexist during all analysed period although one of these, the directly driven process, is the dominant component of the geomagnetic daily variation.

Acknowledgements. The results presented in this paper rely on public data collected at magnetic observatories and available by INTERMAGNET (www.intermagnet.org). We thank the national institutes that support them and INTERMAGNET for promoting high standards of magnetic observatory practice. The authors kindly acknowledge N. Papitashvili and J. King at the National Space Science Data Center of the Goddard Space Flight Center for the use permission of 1-min OMNI data and the NASA CDAWeb team for making these data available. This work is supported by Italian National Program for Antarctic Research (PNRA) Research Project 2013/AC3.08. The elaborated data for this paper are available by contacting the corresponding author (paola.demichelis@ingv.it). 


\section{References}

Akasofu, S.-I., B.-H. Ahn and G.J. Romick (1983). A study of the polar current systems using the IMS meridian chains of magnetometers, Space Sci. Rev., $36,337$.

Campbell, W. (1989). The regular geomagnetic field variations during solar quiet conditions, Geomagnetism, vol. 3, Elsevier, New York.

Chen, G.-X., et al. (2007). Statistical characteristics of the day-to-day variability in the geomagnetic $S_{q}$ field, J. Geophys. Res., 112, A06320; doi:10.1029/ 2006JA012059.

Clauer, C.R., and Y. Kamide (1985). DP1 and DP2 current systems for the March 22, 1979 substorms, J. Geophys. Res., 90, 1343.

Consolini, G., R. Tozzi and P. De Michelis (2009). Complexity in the sunspot cycle, Astron. Astrophys., 506, 1381.

Davis, T.N., and M. Sugiura (1966). Auroral electrojet activity index $\mathrm{AE}$ and its universal time variations, J. Geophys. Res., 71 (3), 785.

De Michelis, P., R. Tozzi and A. Meloni (2009). On the terms of geomagnetic daily variation in Antactica, Annales Geophysicae, 27, 2483.

De Michelis, P., R. Tozzi and G. Consolini (2010). Principal components's features of mid-latitude geomagnetic daily variation, Annales Geophysicae, 28, 2213.

Feldestein, Y.I., and A.N. Zaitzev (1967). Magnetic field variations at high latitudes on quiet days in summer during the IYG, Geomagn. Aeron., 7, 160.

Friis-Christensen, E., Y. Kamide, A.D. Richmond and S. Matsushita (1985). Interplanetary magnetic field control of high-latitude electric field and currents determined from Greenland magnetometer data, J. Geophys. Res., 90, 1325.

Frynberg, E.B. (1975). Separation of the geomagnetic field into a normal and anomalous part, Geomagn. Aeron., 15, 117.

Golovkov, V.P., N.E. Papitashvili, Y.S. Tyupkin and E.P. Kharin (1978). Separation of geomagnetic field variations on the quiet and disturbed components by the MNOC, Geomagn. Aeron., 18, 342.

Kawasaki, K., and S-I. Akasofu (1967). Polar solar daily geomagnetic variations on exceptionally quiet days, J. Geophys. Res., 72 (21), 5363.

Langel, R.A. (1987). The main field, In: J.A. Jacobs (ed.), Geomagnetism, Academic, San Diego, Calif., vol. 1, 249.

Nagata, T., and S. Kokubun (1962). A particular geomagnetic daily variation $S_{q}^{p}$ in the polar regions on geomagnetically quiet days, Nature, 195, 555.

Olsen, N. (1997). Geomagnetic tides and related phe- nomena, In: W.H. Zuern and H.G. Wenzel (eds), Tidal Phenomena, Springer, Berlin, 261 p.

Pushkov, A.N., E.B. Frynberg, T.A. Chernova and M.V. Fiskina (1976). Analysis of the spacetime structure of the main geomagnetic field by expansion into natural orthogonal components, Geomagn. Aeron., 16, 196.

Richmond, A.D. (1995). Ionospheric electrodynamics, In: H. Volland (ed.), Handbook of atomospheric electrodynamics, CRC Press, Boca Raton, 249.

Rotanova, N.M., N.E. Papitashvili and A.N. Pushkov (1982). Use of natural orthogonal components to distinguish and analyse the 60-yr geomagnetic field variations, Geomagn. Aeron., 22, 821.

Sun, W., W.-Y. Xu and S.-I. Akasofu (1998). Mathematical separation of directly driven and unloading components in the ionospheric equivalent currents during substorms, J. Geophys. Res., 103, 1695.

Sun, W., W.-Y. Xu and S.-I. Akasofu (2000). An improved method to deduce the unloading component for magnetospheric substorms, J. Geophys. Res., 105, 13131.

Xu, W.-Y., M.-L. Zhang, Y.-F. Lin and X.-P. Zeng (1990). Analysis on structure of the variable geomagnetic fields at middle and low latitudes, Chinese J. Geophys., 33, 12.

Xu, W.-Y., and Y. Kamide (2004). Decomposition of daily geomagnetic variations by using method of natural orthogonal component, J. Geophys. Res., 109, A05218; doi:1029/2003JA010216.

Corresponding author: Paola De Michelis, Istituto Nazionale di Geofisica e Vulcanologia, Rome, Italy; email: paola.demichelis@ingvit.

(C) 2015 by the Istituto Nazionale di Geofisica e Vulcanologia. All rights reserved. 